\title{
Application of CART3D to Complex Propulsion-Airframe Integration with Vehicle Sketch Pad
}

\author{
Andrew S. Hahn ${ }^{1}$ \\ NASA Langley Research Center, Hampton, VA, 23681
}

\begin{abstract}
Vehicle Sketch Pad (VSP) is an easy-to-use modeler used to generate aircraft geometries for use in conceptual design and analysis. It has been used in the past to generate metageometries for aerodynamic analyses ranging from handbook methods to Navier-Stokes computational fluid dynamics (CFD). As desirable as it is to bring high order analyses, such as CFD, into the conceptual design process, this has been difficult and time consuming in practice due to the manual nature of both surface and volume grid generation. Over the last couple of years, VSP has had a major upgrade of its surface triangulation and export capability. This has enhanced its ability to work with Cart3D, an inviscid, three dimensional fluid flow toolset. The combination of VSP and Cart3D allows performing inviscid CFD on complex geometries with relatively high productivity. This paper will illustrate the use of VSP with Cart3D through an example case of a complex propulsion-airframe integration (PAI) of an over-wing nacelle (OWN) airliner configuration.
\end{abstract}

\section{Nomenclature}

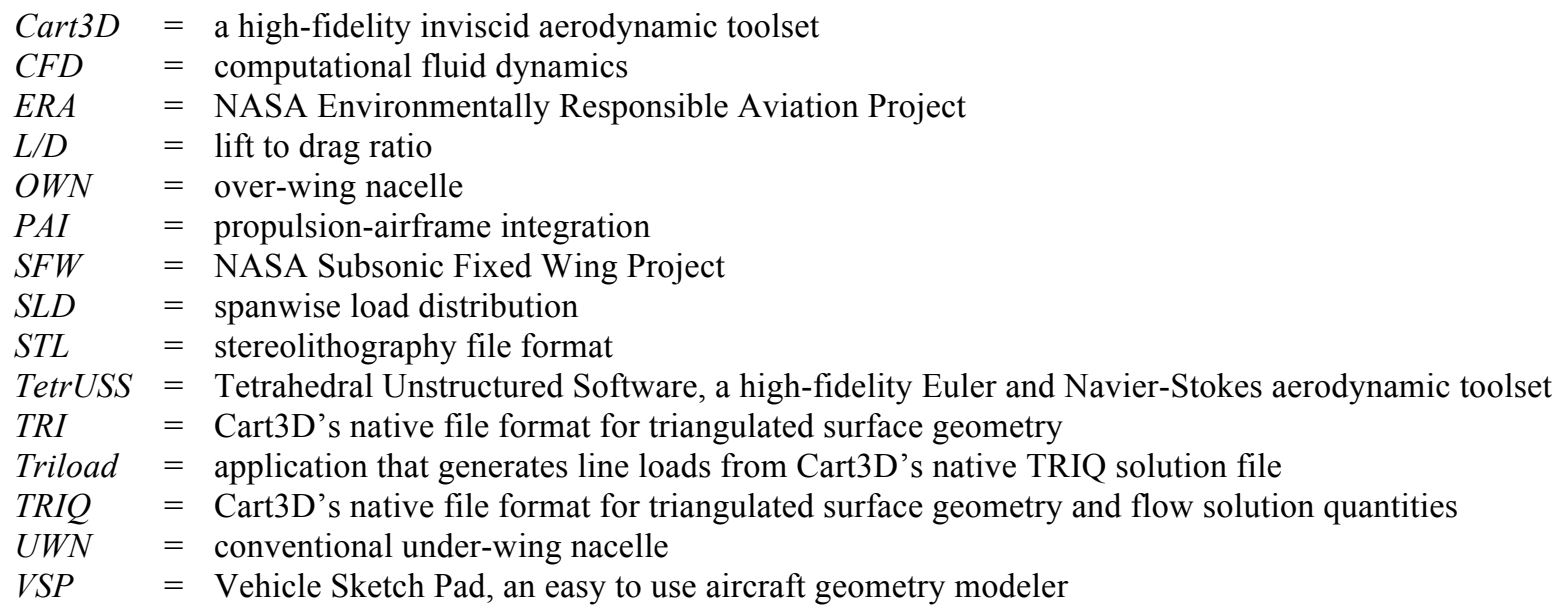

\section{Introduction}

$\mathrm{H}$ ISTORICALLY, conceptual aircraft design has been limited to analyses that are of low order so as to take advantage of the productivity that automation may provide. This has meant that the most appropriate analyses were regressions of historical data, using relatively few degrees of freedom, and being dependent upon low diversity data sets. This works extremely well when analyzing configurations that are within historical norms, using extrapolations associated with technologies whose expected impacts may be applied in terms of changes to the norm. Indeed, this approach has many advantages, not the least of which is that it is possible to have accurate predictions despite having relatively little information describing the configuration. Not all of the design details are required because the assumption is that the data set is taken from aircraft that have had a tremendous amount of effort expended on them and that they represent the best that is possible within the limits of the design choices available at the time.

\footnotetext{
${ }^{1}$ Aerospace Engineer, Aeronautics Systems Analysis Branch, Mail Stop 442, AIAA Member 193989.

1

American Institute of Aeronautics and Astronautics
} 
Unfortunately, this approach locks the designer into a subset of the design space that is well trodden, and it may not contain the best possible configuration, particularly if there has been a major change in the requirements set or the availability of some new technology. The desire to achieve radical improvements in multiple metrics has prompted a greater interest in being able to investigate unusual aircraft configurations. These unusual configurations may be better solutions given new technologies, such as ultra high bypass ratio engines, or new requirements, such as reduced community noise.

In order to investigate unusual configurations, it becomes necessary to expand analysis methods to higher orders. These higher order analyses have the advantage of being sensitive to many more degrees of freedom, allowing the designer to make choices that are outside of the norm of the historical database. The disadvantage of these analyses is that the same sensitivity to more degrees of freedom increases the amount of information that the designer needs to provide. Often, the increase in information required can be large, creating whole new sub-design problems that reduce productivity dramatically. Part of this productivity reduction is unavoidable due to the very nature of the problem, in that a design effort that was implicit in the historical database now needs to be performed explicitly by the designer. The other part of the productivity reduction may be avoidable to some extent through the characteristics of the analysis methods chosen.

A good example of the reduction of productivity through the use of higher order analyses is the use of computational fluid dynamics (CFD) in conceptual design. When employing handbook methods, the designer needed only to provide basic geometric information such as wing plan form, wetted areas, thickness ratios, and a general class of airfoil type. Optimization was useful because of the few design variables required, automatic calculation of changed geometries, and high speed of solution execution. Higher order methods require that the wing be described in much greater detail. Often, these details are not publicly known for an existing aircraft or have not been optimized on an original configuration. In either case, the designer loses the benefit of the effort expended by others and must now provide the effort himself. This task can be made all the more difficult by the simple fact that an unusual configuration is by its very nature unexplored. This means that many of the rules of thumb that the designer may have built up through familiarity with the conventional configurations may be of little use, or worse, may be detrimental. The unusual configuration must be designed from scratch to a level of detail and sophistication that is generally not done in conceptual design. The difficulty of the task is only compounded when generating and analyzing the geometry requires expertise in complex software along with significant manual effort and long execution times.

Both Vehicle Sketch Pad (VSP) and Cart3D are designed to reduce the burden associated with generating a representative aircraft geometry and performing inviscid aerodynamic analysis. ${ }^{1,2}$ Also, recent developments in VSP's triangulation and export capabilities make coupling to Cart3D easier than ever.

The following will illustrate the use of VSP with Cart3D through an example case of a complex propulsionairframe integration (PAI) of an over-wing nacelle (OWN) airliner configuration.

\section{Using VSP With CFD}

NASA projects, such as Environmentally Responsible Aviation (ERA) and Subsonic Fixed Wing (SFW), are seeking to develop technologies and configurations for future airliners that will have radically improved performance as measured by fuel economy, community noise, and emissions metrics. Whereas these metrics have always been important, the much stronger emphasis adopted by these projects is forcing airliner designers to look at technologies and configurations that would not have been viable before. In particular, the fuel economy and community noise metrics are driving engine designs to ultra-high bypass ratios. These ultra-high bypass ratios make the resulting engine diameter exceptionally large when compared to current engines. This characteristic makes the conventional PAI choice of suspending the engines under a low wing less attractive. One potentially attractive PAI option is to move the engine from under the wing to over the wing. There are many advantages to integrating a large diameter engine over the wing, but there is one major disadvantage. This disadvantage is the fact that all attempts to do so have resulted in unacceptably greater drag, particularly during transonic cruise. Since the historical database would only consist of aircraft or wind tunnel tests that had these higher drag characteristics, the common low order conceptual design approach will always evaluate OWNs unfavorably. Trying to find an OWN configuration that performs as well as the conventional under-wing nacelle (UWN) PAI choice requires the use of higher order aerodynamic methods. It is this necessity that is driving the use of VSP with CFD. 


\section{A. Previous VSP CFD Experience}

The current OWN configuration of interest had been investigated more than 10 years ago using a predecessor of VSP with both full potential and Euler CFD codes. ${ }^{3}$ Whereas these early results had hinted that there was a compressibility phenomenon that might be exploitable for reducing transonic drag, there were problems with the geometry and flow solutions that made the results far from conclusive. In an attempt to build on these earlier results, Geoffrey Hill performed a study in 2007 using an early version of VSP providing surfaces to the TetrUSS aerodynamic toolset. ${ }^{4-6}$ His results showed that there was a reduction in transonic drag due to the unusual flow in the channel formed by the fuselage, wing, and nacelle

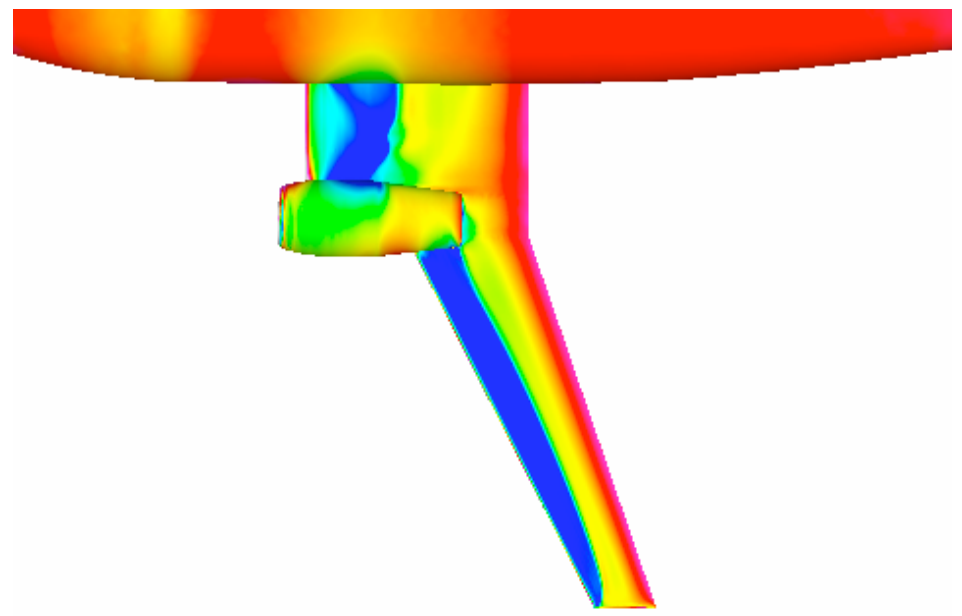

Figure 1. Hill TetrUSS CFD solution.

(Fig. 1). Unfortunately Hill also found that the drag in the nacelle region more than offset the reduction in the channel. We felt that additional effort in modifying the nacelle geometry would be beneficial; however, the difficulty of generating the grids necessary for additional solutions was such that further investigation could not be done in the allotted time.

This experience highlighted the need to have a CFD analysis that is easier to use and more productive. A major enhancement to Cart3D rekindled interest in using this inviscid fluid flow toolset. Cart3D was always aimed at providing automated grid generation for increased productivity; however, it was the enhancement of an adjointbased optimized volume grid refinement that made Cart3D particularly attractive for the transonic flow conditions that this concept requires. ${ }^{7}$ At about the same time, the surface triangulation capability of VSP had been significantly enhanced, improving interoperability with Cart3D.

\section{B. VSP Surface Triangulation}

Cart3D only needs a water-tight triangulated surface in order to automatically generate the Cartesian volume grid necessary for calculating flow solutions. VSP has always had the ability to triangulate and export water-tight surfaces. This original method of triangulation, called CompGeom, is performed by simply laying diagonals into the existing structured quadrilateral surface representation. The designer has good control over the resolution and aspect ratios of the quadrilaterals through VSP's tessellation controls. Whereas the CompGeom method of triangulation worked well for a single component, when more than one component is intersected, the resulting triangulation is unsuitable for most CFD analyses. This prompted the development of VSP's High Quality triangulated surface meshing capability. The main advantages of VSP's High Quality meshing are that the designer has much tighter control over the distribution and size of the surface triangles, as well as ensuring that all of the triangles will be of acceptable aspect ratio, even with multiple components being intersected.

Either method of surface meshing is acceptable to Cart3D, and the designer is free to choose the method that suits the problem at hand best. Both methods are able to export in the stereolithography (STL) file format, which Cart3D imports, and Cart3D's native triangulated surface geometry (TRI) file format.

Surface meshing for Cart3D is somewhat different from that done for most tetrahedral based CFD analyses. Most tetrahedral volume meshes are grown from the surface triangulation, so the surface triangulation controls the volume mesh distribution and the aspect ratios of the tetrahedra directly. This is what prompted VSP's High Quality mesh capability, giving fine control of the surface mesh to the designer. Whereas this fine control allows the triangulation to be distributed in such a way that the representation of the surface may be done efficiently, this is less important with Cart3D. What is important to Cart3D is simply that the triangulation accurately reflects the original surface. The resolution and aspect ratios of the surface triangles do not influence the resolution or aspect ratios of the volume mesh directly. In fact, Cart3D's volume mesh is made up of cubes, so the aspect ratio is never an issue. With the enhancement of the adjoint-based grid refinement feature, Cart3D's volume mesh resolution is driven by the sensitivity of the solution to the mesh topology, not the resolution of the surface triangles. Given this, we have settled on the process of using the tesselation controls to produce very fine CompGeom surface meshes. While this does produce relatively large surface files, these are still small when compared to the other files that are produced by Cart3D and surface accuracy is assured, no matter how the volume mesh is defined. 
Generally, the easiest procedure for exporting geometry to Cart3D is to tessellate each component as desired, show all of the wetted components in the VSP viewer window, select CompGeom from the Geom pulldown menu, and then export the resulting Mesh component in Cart3D's native TRI file format. Cart3D is then able to operate directly on this geometry.

\section{Initial VSP Geometry Definition}

VSP gives the designer access to several component types with which to build a model. These components are defined parametrically using familiar terms to aerospace engineers. Since these components are defined by a relatively small number of parameters, typical aircraft shapes are easily produced. These parametric definitions may also be expanded as needed to capture more complex geometries. While it is possible to expand these definitions to the point that nearly arbitrary shapes may be represented, this approach defeats the benefits of having a parametrically defined geometry, and so is generally considered poor practice.

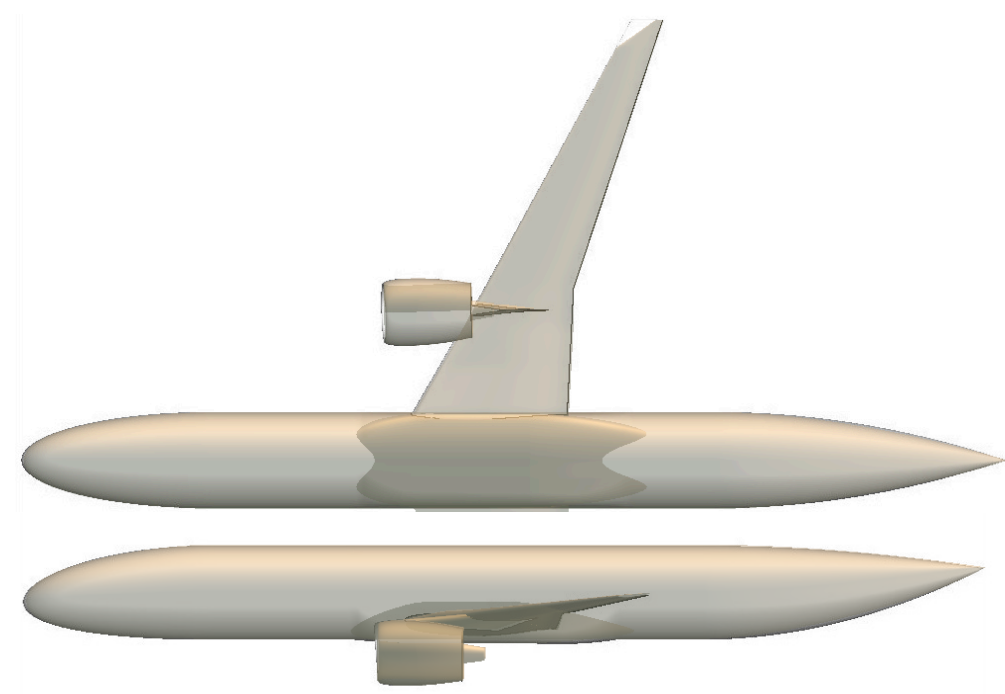

Figure 2. Reference UWN Configuration Comparison.

Often, an acceptable representation may be had with relatively few parameters. In this particular case, we received a TRI file of a conventional UWN configuration that we wanted to use as a starting point, as well as the parameters that were used to create the original CAD geometry. Using this information, an acceptable representation of the UWN reference was created. Figure 2 shows the VSP model overlaying the original translucent TRI file surface. The model is built out of only three component types; fuselage, multi-section wing, and duct. There are several small differences, most notably the lack of scarfing on the engine inlet, the shape of the fuselage fairing, and the wing tip, which is sheared. None of these differences was deemed significant, and the fuselage, fairing, and outboard wing planform were carried on to the OWN configuration.

The major differences between the UWN and OWN configurations are the wing planform and the nacelle shape. Since the wing planform of the original TRI file didn't have sheared tips, we decided to go with the simpler rectangular tips, and modify the inboard section for the OWN integration. Whereas the UWN has a separate flow nacelle with a short fan duct, the OWN must have a long duct. The original duct shape was extended while maintaining the maximum diameter as well as inlet and nozzle areas.

It became immediately obvious that we could no longer use the duct component for the nacelle, as it was limited to a body of revolution, restricting all of the crossections to being circular. This meant that the lower part of the exhaust nozzle and the upper wing surface formed acute angles, which would produce poor flow characteristics. We then tried the tactic of defining the nacelle as two separate fuselage components, one for the outer mold line
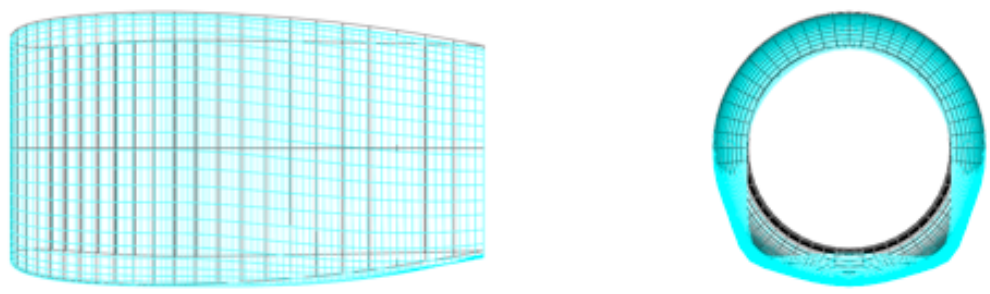

Figure 3. Nacelle Comparison. Duct Component (black) vs. Fuselage Component (blue).

and one for the inner mold line, where the first and last cross sections are identical. This was likely to fail, as it violated the requirement for a fuselage to begin and end at a point in order to ensure that it is water-tight. Much to our surprise, when the CompGeom intersected triangulation was exported in STL format, Cart3D was able to put the pieces together correctly and performed as expected. Initially, this approach did not work with the native TRI format export or the High Quality surface mesh, but VSP will now recognize this case and merge the open components into a single water-tight component. Constructing the nacelle in this way gives many more degrees of freedom, allowing for much more modification than there otherwise could be. This approach allowed the exhaust nozzle to be shaped like a rolled over "D" with the lower part of the nozzle buried in the wing (Fig. 3). For the first configuration, the 


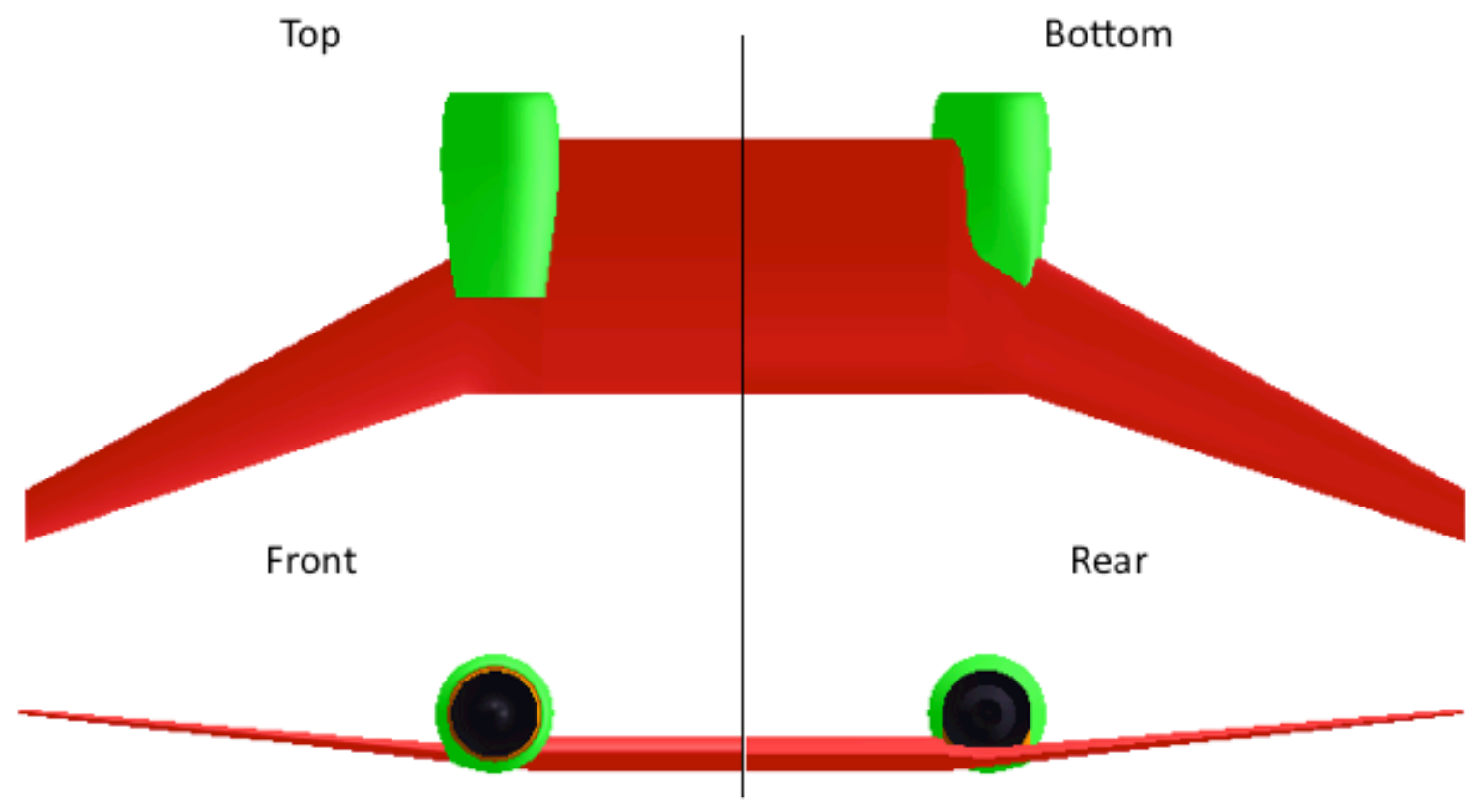

Figure 4. OWN planform with symmetric engine nacelles.

nacelles were left-right symmetric, as they were in Hill's investigation. Figure 4 shows the initial OWN planform with this nacelle definition intersected.

\section{Initial Cart3D Results}

The full aircraft configuration, except tails, was finely tessellated, meshed with CompGeom, and exported as an STL to a Cart3D working directory on a computer cluster. The nacelles were run in a flow through condition and the angle of attack was varied until the design cruise lift coefficient was achieved. This took three runs at approximately 3.5 hours each on a 30 node shared memory cluster computer requiring 11 adaption cycles. Figure 5 shows the Cart3D flow solution for this configuration at a free stream Mach number of 0.78 . The solution shows strong shocks in the channel formed by the fuselage, inboard wing, and nacelle. The solution also

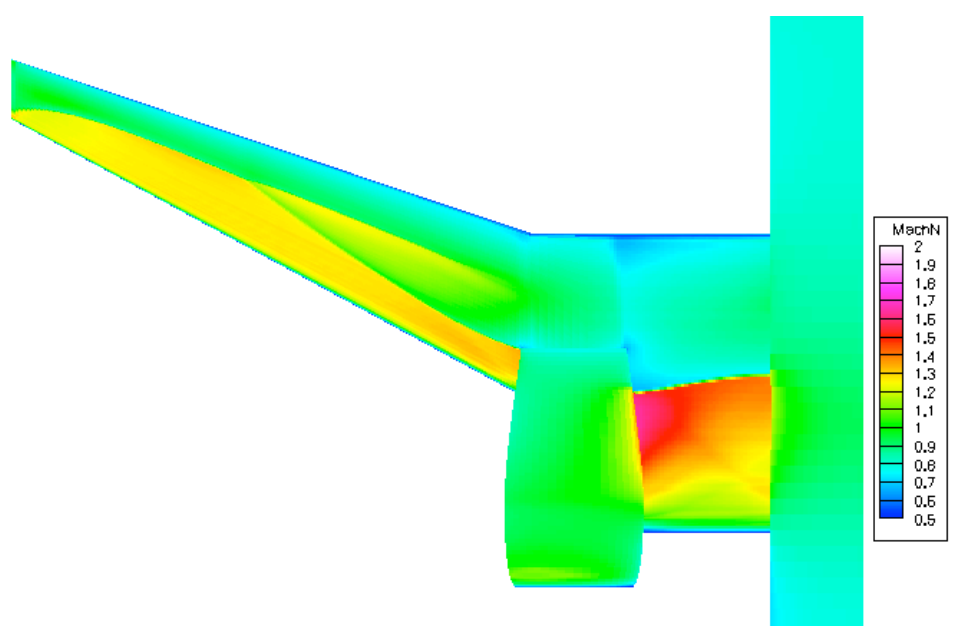

Figure 5. Initial OWN06a Cart3D flow solution.

shows a moderate shock just outboard of the nacelle that disrupts the airfoil pressure distributions out to approximately half of the outboard wing section. Since the outboard airfoil sections are of supercritical design, the airflow attempts to assume the classic aft shock pressure distribution and the flow reaccelerates, culminating in a second weak shock. This solution estimated a relatively low inviscid lift to drag ratio (L/D) of 27, indicating that it is a poor design.

This result illustrates the designer's dilemma mentioned earlier, associated with bringing high order analyses into conceptual design. High order analysis is able to provide important information about unusual configurations that are outside of the historical database; however, these analyses only evaluate the specific design supplied, creating the need to determine the best possible design. 


\section{E. Triload Design Support}

As important as the flow solution is, it is insufficient for guiding the designer's decisions. The designer needs information from the solution provided in such a way that he can then make informed decisions about which changes in the geometry are likely to improve the configuration's performance. The previously mentioned OWN studies had as a fundamental constraint that all final solutions were to have as much as possible the same spanwise load distribution (SLD). Holding SLD constant is important because the configuration's induced drag is determined by it. Whereas there is debate as to which SLD is best for this class of aircraft, the actual shape of the SLD is less important than the variance of the shape between configurations in a comparison study. Since any SLD may be designed in, different SLDs resulting in different induced drags are undesirable distractions.

In order to extract the configuration's SLD from the Cart3D solution file, we obtained the software application Triload from the Chimera Grid Tools toolset. ${ }^{8}$ Triload is a very flexible tool that generates line loads from Cart3D's native TRIQ solution file. Once set up, Triload is generally able to perform the SLD calculations as the design changes without manual intervention.

Figure 6 shows Triload extractions of lift and drag as a function of span along with equivalent-lift parabolic and elliptical SLDs for use as guides. We decided to use the elliptical SLD to guide our design changes. The independent axis represents the local spanwise location and is in units of feet. From zero to 6.2 feet is occupied by the fuselage. There is very little that can be done in this region, particularly as fuselages don't really generate lift in an inviscid solution as they would in reality. Whatever lift is there is mostly bleed over from the wing. From 6.2 to 14.6 feet is the inboard wing section, from 14.6 to 23.8 feet is the engine nacelle, and from 23.8 to 56.6 feet is the outboard wing.

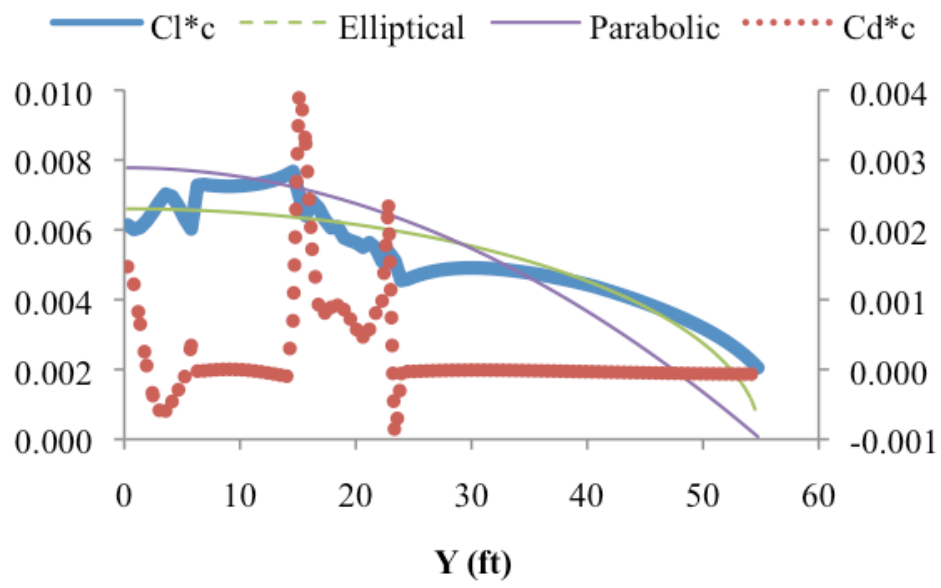

Figure 6. Initial OWN06a SLDs.

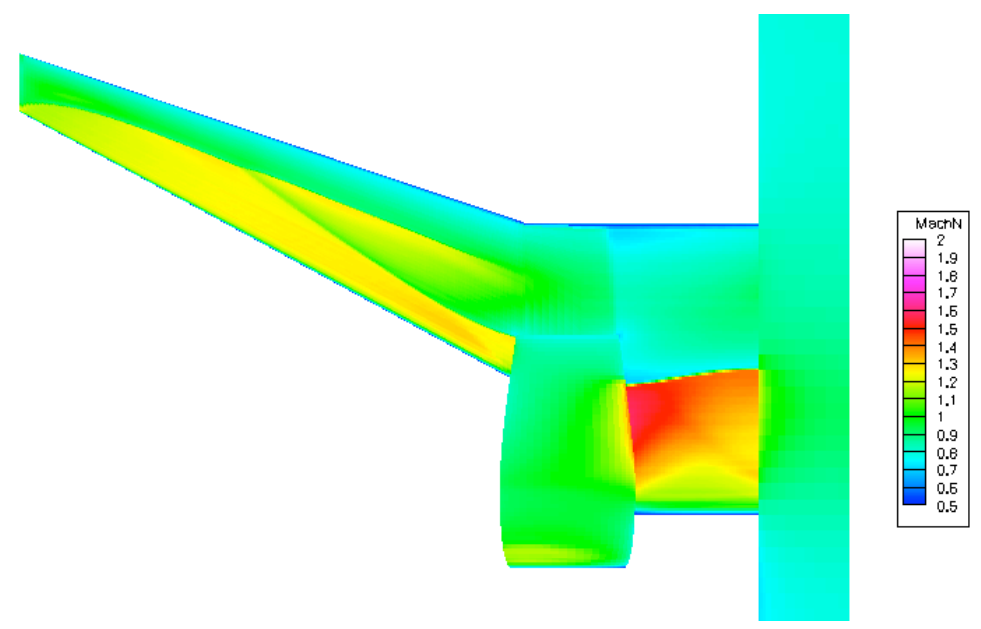

Figure 7. Twisted OWN06b Cart3D flow solution.

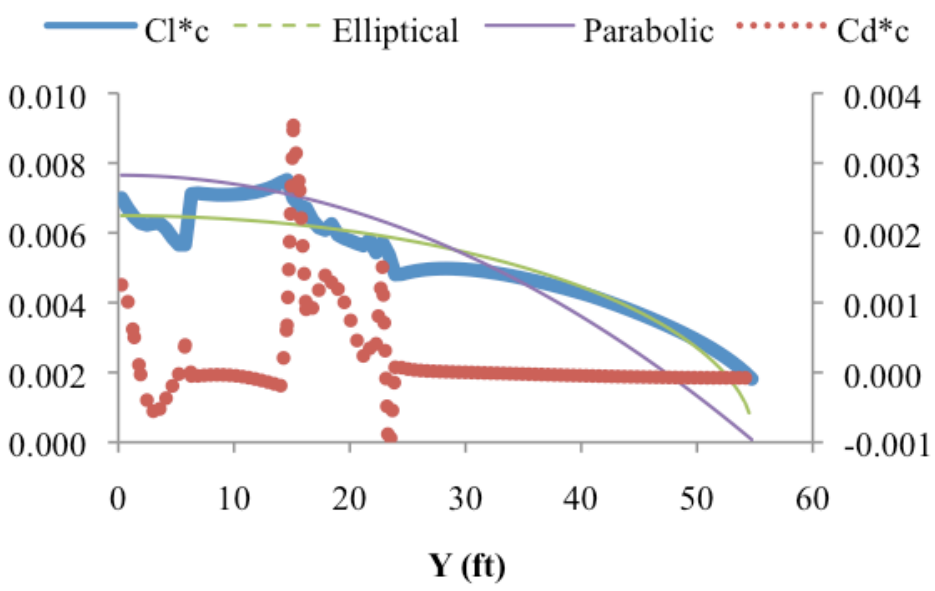

Figure 8. Twisted OWN06b SLDs. 


\section{F. First Geometry Modification}

It is clear from Figure 6 that the outboard wing is carrying too much lift, so the first modification to the geometry was to change the outboard wing's twist from 1.5 to 2.5 degrees. The previous process of triangulating, exporting, and flow solving was followed, resulting in the new solution (Fig. 7) and SLDs (Fig. 8). This result looks better than the initial result, and this qualitative judgment is corroborated by an inviscid L/D of 33, a significant improvement of 22 percent. Still, the major feature of the lift distribution is the large drop in lift across the nacelle location. A great deal of vorticity is being shed, making the induced drag still unacceptable. Hill observed a similar behavior and decided to tailor wing twist locally to match the elliptical guide SLD. This is a perfectly logical approach; however, this would also result in unusually high local wing twist angles, which are undesirable.

\section{G. Second Geometry Modification}

We decided to try nacelle tailoring instead of wing twist. Since the nacelles were constructed from fuselage components, the parameters were already available to easily camber the nacelle in the $\mathrm{X}-\mathrm{Z}$ plane. The only parametric changes needed were, to give the nacelle's last cross section a Y offset and to change the tangent angles on each side to something that looked reasonable. Figure 9 is the Cart3D flow solution and the cambered nacelle shape is clearly visible. The difference between Figures 7 and 9 is striking. The shocks in the inboard wing section are significantly lower and closer to being uniform. The shock on the outside of the nacelle has been strengthened, but even so, the inviscid L/D improved to 36 , or by 9 percent. Figure 10 shows the SLD, which is greatly improved. The wing's inboard and outboard lift distributions are nearly where they should be. The peaks at the sidewalls of the nacelle have swapped directions, indicating that the nacelle has been cambered more than needed. This may also explain why there is a significantly stronger shock on the
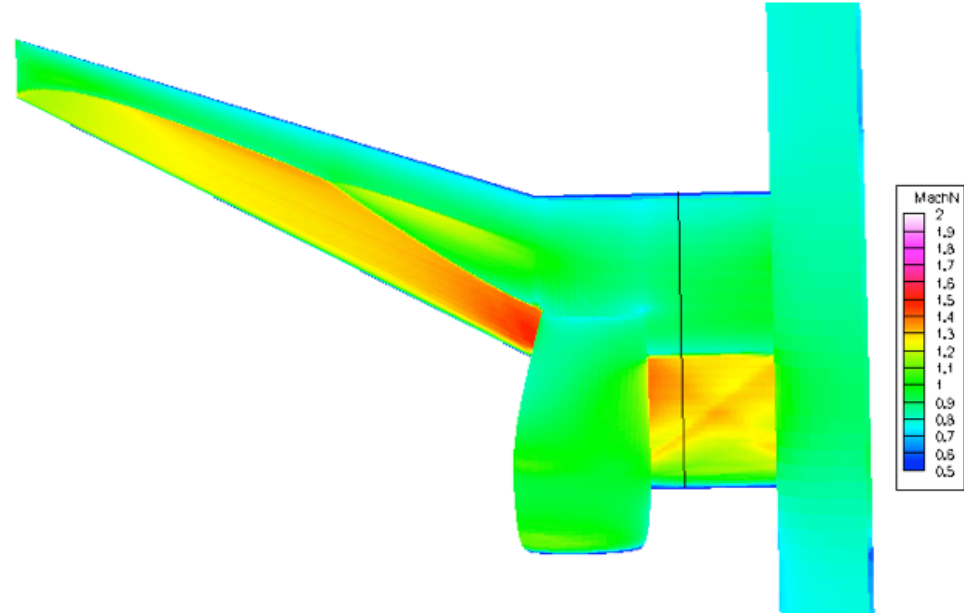

Figure 9. Cambered nacelle OWN06c Cart3D flow solution.

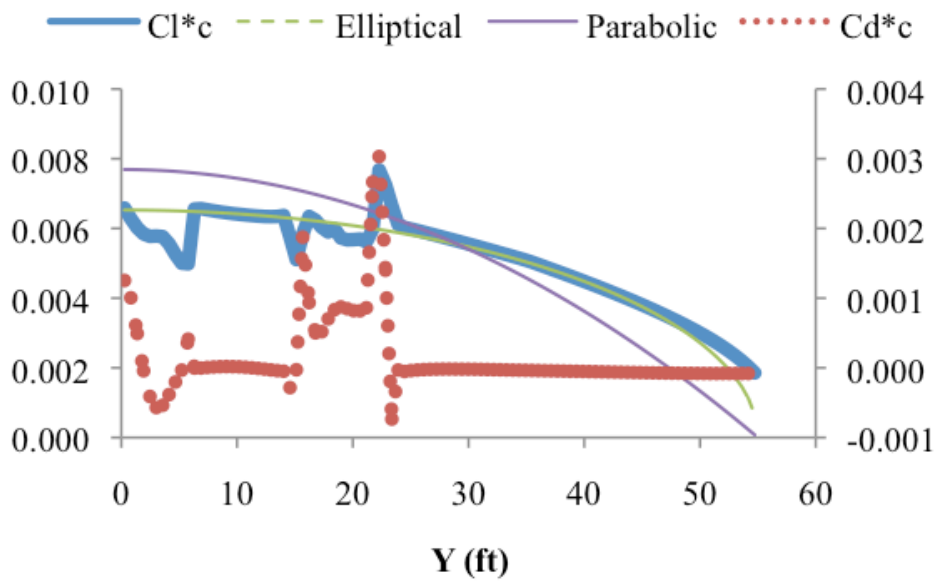

Figure 10. Cambered nacelle OWN06c SLDs. outside of the nacelle.

\section{H. Engine Simulation Modification}

Since this is a PAI example, it would seem prudent to consider simulating the engine's propulsive influence on the aircraft's aerodynamics at some point. So far, all of the aerodynamic solutions have been performed with unpowered, flow through nacelles. Cart3D does have the ability to simulate the influence of an engine. ${ }^{9}$ The previous model was modified to enable engine simulation to see if there would be a significant change in the flow field. Performing this kind of analysis significantly complicates both the modeling and the aerodynamic analysis. We felt that it was important to try the engine simulation at this point to see whether or not it would be possible to continue geometry refinement using the simpler flow through scheme. If the engine's influence dramatically changes the aerodynamic solution, then we would be forced to continue refinement with the more difficult engine simulation. 
After some experimentation, we found that the best way to model the simulated engine was to duplicate the inner fuselage component, then turn one of them into a short inlet, terminating approximately at the engine fan face location and then turn the other into a nozzle, starting a short distance in from the exit (Fig. 11). In both cases, the components block what was originally a flow path. Since VSP tags all of the
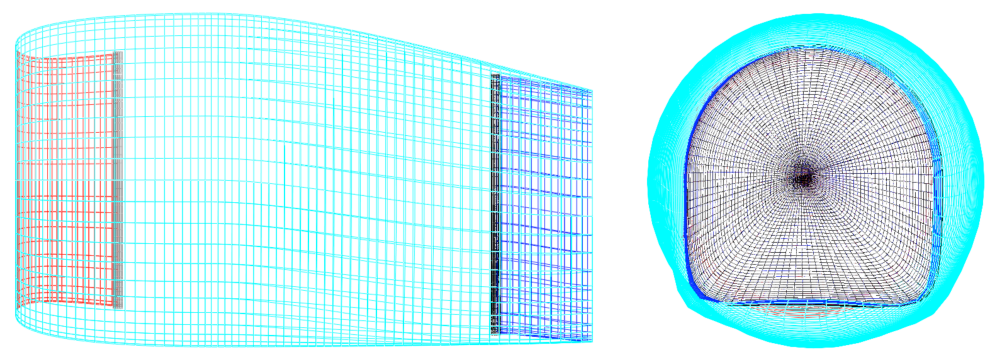

Figure 11. Simulated engine nacelle model.

triangles of a component with the same number, it is not possible to assign different boundary conditions to different parts of a component. The way around this is to create two new fuselage components that, for lack of a better description, are called hockey pucks. These hockey pucks intersect the inlet and nozzle in such a way that they now provide the surface triangles representing the flow areas. Since these are two different components, their surface triangles have unique tags that then can have special boundary conditions applied to them by Cart3D. There are a number of changes that have to be made to the scripts that run Cart3D as well as the burden of determining what the inlet and nozzle boundary conditions must be. Add to this the increased difficulty of force bookkeeping and it becomes very attractive to avoid performing powered simulations for as long as possible.

Figure 12 shows the Cart3D flow solution for a simulated 1.35 fan pressure ratio engine. This solution did converge, but the convergence was less robust and the solution required approximately twice the amount of time to complete, compared to the previous solution. Whereas there are certainly differences, the basic character of the solution is approximately the same. In particular, the wing shocks all look a little stronger, which is certainly due to the influence of the engine's flow; however, a check of the lift coefficient showed that it is somewhat higher, indicating that the angle of attack should be reduced. Reducing the angle of attack to achieve the design lift coefficient

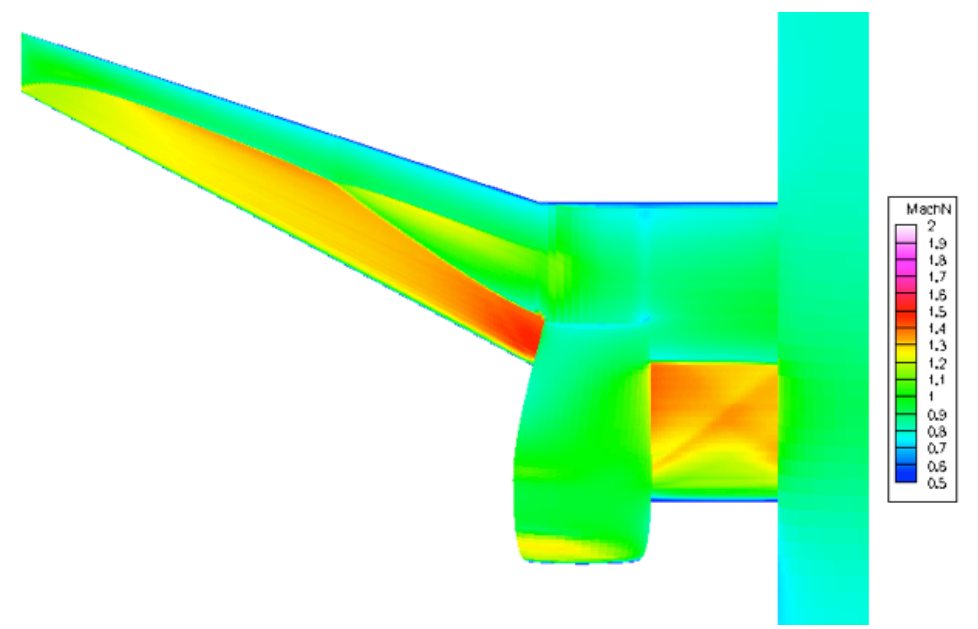

Figure 12. Engine OWNO6c+ Cart3D flow solution.

should reduce the shocks somewhat. Despite the engine's propulsive effect having a clear influence on the aerodynamic solution, it is reasonable to continue performing aerodynamic refinements using the simpler, more robust, and faster flow through scheme.

\section{Future Work}

There are a number of design options that need to be explored to see if further aerodynamic improvements can be made. While there is work remaining on this configuration, we now have a set of tools with which to understand, evaluate, and make changes. Up until now, these changes have all been performed manually, relying on interpretations of the surface pressures and load distributions, which are indirect indicators of the objective, namely minimizing drag at a constant lift coefficient.

Recently, a beta version of the Cart3D Adjoint-Based Design Framework has been released. This framework promises to provide an automated capability of optimizing a geometry with respect to a specified objective. ${ }^{10}$ This capability would calculate the gradient of geometric parameters with respect to the objective directly and automatically. Our goal is to enhance VSP's features to work with the design framework seamlessly. If successful, this capability would dramatically improve the productivity of using high order CFD in the conceptual design phase. 


\section{Conclusion}

The OWN PAI design task has proven to be an excellent stress test of the ability of VSP to model and export complex aircraft geometry for analysis using Cart3D CFD. Encountering unexpected problems has revealed items in need of improvement as well as prompting the creation of new and unanticipated features. This effort has resulted in a set of tools that improves the productivity of using high order, inviscid CFD analysis in the conceptual design phase. This capability frees the conceptual aircraft designer from the constraint of a historical configuration database, while reducing the burden that is associated with that freedom. The current versions of VSP, Triload, and Cart3D support manual geometric manipulation and aerodynamic evaluation, and we are excited by the potential of coupling these tools together with the automated adjoint-based design framework in the future.

\section{Acknowledgments}

I would like to thank J.R. Gloudemans for continuing to develop Vehicle Sketch Pad, making it the excellent tool that it is. I would also like to thank Dr. Robert McDonald for his support and patience over the last year that this work was being performed. There were a number of problems encountered along the way and I would have accomplished far less without having access to his knowledge, skill and effort. Last, I would like to thank the support that NASA's Environmentally Responsible Aviation Project has given.

\section{References}

${ }^{1}$ Hahn, A. S., "Vehicle Sketch Pad: A Parametric Geometry Modeler for Conceptual Aircraft Design," AIAA-2010-657, AIAA, Reston, VA, 2010.

${ }^{2}$ Cart3D, Inviscid Aerodynamics Analysis, Software Package, Ver. 1.4, NASA Ames Research Center, Moffett Field, CA, URL: http://people.nas.nasa.gov/ aftosmis/cart3d/ 2011.

${ }^{3}$ Kinney, D. J., Hahn, A. S., and Gelhausen, P. A., "Comparison of Low and High Nacelle Subsonic Transport Configurations," AIAA-1997-2318, AIAA, Reston, VA, 1997.

${ }^{4}$ Frink, N. T., Pirzadeh, S. Z, Parikh, P. C., Pandya, M. J., and Bhat, M. K., "The NASA Tetrahedral Unstructured Software System," The Aeronautical Journal, Vol. 104, No. 1040, October 2000, pp. 491-499.

${ }^{5}$ Hill, G., and Kandil, O., "Aerodynamic Investigations of an Advanced Over-the-Wing Nacelle Transport Aircraft Configuration," AIAA-2007-670, AIAA, Reston, VA, 2007.

${ }^{6}$ Hill, G., Kandil, O., and Hahn, A. S., "Aerodynamic Investigations of an Advanced Over-the-Wing Nacelle Transport Aircraft Configuration," Journal of Aircraft, Vol. 46, No. 1, 2009, pp. 25-35.

${ }^{7}$ Nemec, M., Aftosmis, M.J., and Wintzer, M., “Adjoint-based adaptive mesh refinement for complex geometries,” AIAA2008-0725, AIAA, Reston, VA, 2008.

${ }^{8}$ Triload, Generation of line loads from surface triangulation grid and solution file, Software Package, Ver. 1.0e, NASA Ames Research Center, Moffett Field, CA, URL: http://people.nas.nasa.gov/ wchan/cgt/doc/triload.html 2011.

${ }^{9}$ Pandya, S. A., Murman, S. M., and Aftosmis, M. J., "Validation of inlet and exit boundary conditions for a Carteisan method," AIAA-2004-4837, AIAA, Reston, VA, 2004.

${ }^{10}$ Nemec, M., Aftosmis, M.J., and Wintzer, M., "Parallel Adjoint Framework for Aerodynamic Shape Optimization of Component-Based Geometry,” AIAA-2011-1249, AIAA, Reston, VA, 2011. 